\title{
Extra and Intra-articular Synovial Chondromatosis
}

\author{
Rajesh Kumar Chaudhary, 'Bibek Banskota, Saroj Rijal,' Ashok Kumar Banskota' \\ 'Department of Orthopaedics and Traumatology, B \& B Hospital, Kathmandu ${ }^{2}$ Hospital and Rehabilitation Center for Disabled \\ Children (HRDC), Kavre, Nepal.
}

\section{ABSTRACT}

Synovial chondromatosis is not so rare intra-articular condition secondary to synovial metaplasia, that affects the knee joint. Extra-articular synovial chondromatosis however is an extremely rare condition that usually involves the synovial sheath or bursa of the foot or hand.

We present two cases of synovial chondromatosis, one intra and one extra-articular. The first case was a 25 year old lady who presented with pain, swelling and restricted range of motion of left knee and was found to have an intra-articular synovial chondromatosis which was treated successfully by joint debridement. The second case was that of a 22 year old man who presented with right knee pain and was diagnosed to have an extra-articular synovial chondromatosis of his right medial hamstring tendon sheath, excision of which resulted in complete relief of symptoms.

Keywords: extra-articular, intra-articular, metaplasia, rare, synovial chondromatosis.

\section{INTRODUCTION}

Synovial chondromatosis is a rare condition in which islands of metaplastic cartilage develop within the synovium. ${ }^{1}$ It is usually monoarticular, commonly affects the knee joint, ${ }^{1-4}$ is twice as more common in men than in women ${ }^{2,5}$ usually presenting in their third to fifth decade of life. ${ }^{5}$ The occurrence rate is one per 100,000. ${ }^{3}$ Extra-articular osteochondromatosis is extremely rare with few published reports in the literature. ${ }^{2}$ There are only four published cases of extra-articular synovial chondromatosis in prepubescent children. ${ }^{3}$ It arises from the synovium of tendon sheaths and bursae. ${ }^{2}$ Extraarticular synovial chondromatosis is usually solitary ${ }^{7,8}$ and mostly involves the joints of the hands and feet. ${ }^{1,2,7}$ The cause of synovial chondromatosis is uncertain. ${ }^{2,4,5}$ There is metaplastic transformation of synovial cells into chondrocytes via an unknown stimulus. ${ }^{4,9}$ Histologically, it shows cellular atypia mimicking chondrosarcoma and malignant transformation is known to occur, albeit rarely..$^{5,8}$ The signs and symptoms of articular synovial chondromatosis are non specific. ${ }^{9}$ The presenting symptoms are pain, swelling and loss of joint movement. ${ }^{4}$ Radiologically, synovial chondromatosis is characterised by the presence of multiple rounded loose bodies generally uniform in size and with variable degrees of calcification and/or ossification. ${ }^{2}$ It is thus a benign condition with a strong tendency to eventual resolution. ${ }^{9}$ Surgical excision is warranted for symptomatic lesions and those causing symptoms secondary meniscal tears ${ }^{10}$. In patients with extra-articular bursal pathologies, the preferred surgical treatment is complete excision of involved bursa. ${ }^{4}$ Malignant transformation to chondrosarcoma is known complication but fortunately, rarely seen. ${ }^{2}$

\section{Case I}

A 25 year old lady presented to our center with a history of pain and swelling of her left knee for eight years

Correspondence: Dr. Rajesh Kumar Chaudhary, Department of Orthopaedics and Traumatology, B \& B Hospital, Kathmandu, Nepal, Email: drraju.rajesh@gmail.com, Phone: 9841318158. 
which has worsened for the past five months. She also complained of restricted range of motion of the affected knee which was progressive. There was no history of injury to the knee. On examination, there was swelling and tenderness on the lateral aspect of left knee with joint crepitation. There was significant loss of range of motion with flexion of 100 degrees and extension lag of 40 degrees.

Radiological examination showed radiodense intraarticular loose bodies in medial, lateral and posterior compartments of the knee. (Figure 1)

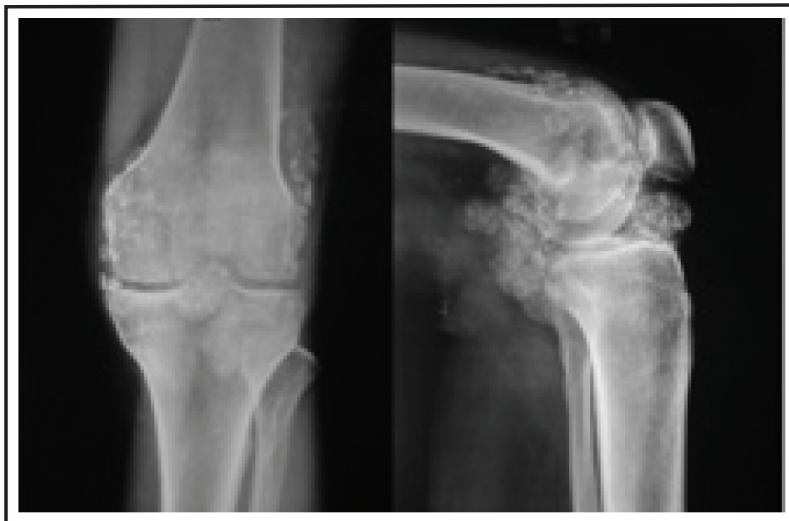

Figure 1. X ray left knee AP and Lateral view showing radiodense intraarticular loose bodies in medial, lateral and posterior posterior compartment of left knee

A diagnostic arthroscopy was carried out through medial and lateral infrapatellar ports. Intraarticular cartilagenous loose bodies were found in the knee joint. A five $\mathrm{cm}$ vertical medial incision was given and the intra-articular loose bodies were removed mostly from anterior compartment of knee joint. The chondromata from the posterior compartment were adherent to the synovial tissue and were not disturbed. (Figure 2) and (Figure 3)

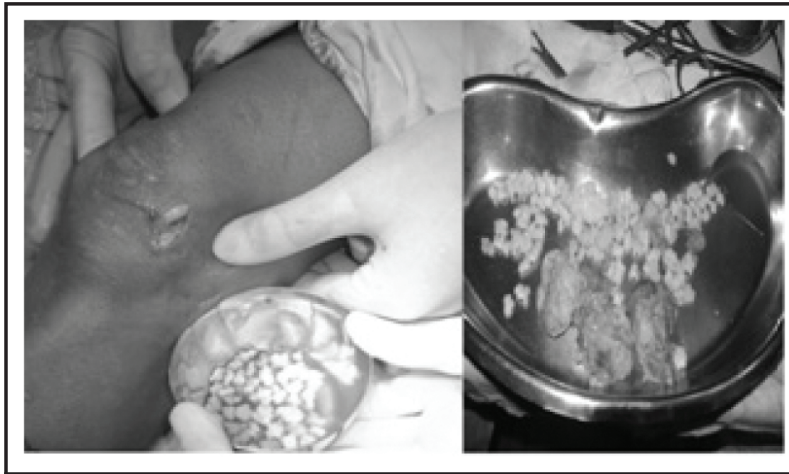

Figure 2. Intraoperative pictures showing cartilagenous loose bodies removed from left knee with synovial tissue

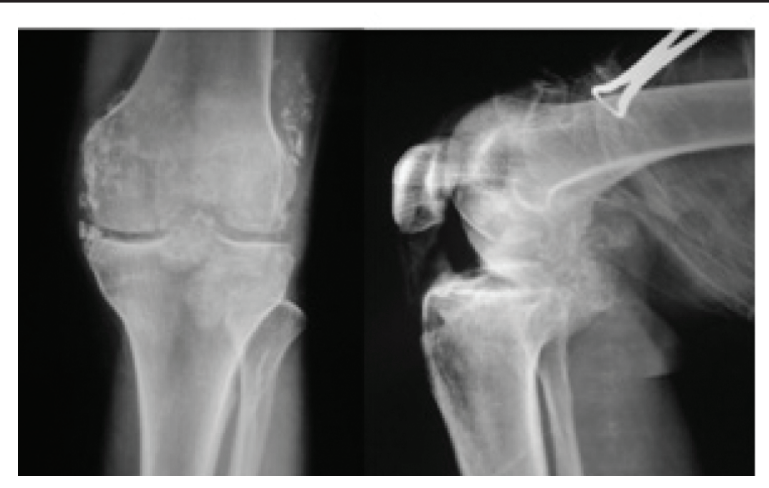

Figure 3. Postoperative $X$ ray of left knee AP and Lateral View showing the remaining radiodense intraarticular loose bodies in superior and posterior aspect with abscence of loose bodies in medial and lateral joint compartment

Post operatively, the patient was pain free with full extension and 100 degrees of knee flexion.

Case II

A 22 year old man presented with complaints of pain in his right knee for three and half years which was increasing in severity. The pain was dull-aching type, associated with some quadriceps wasting but there was no associated swelling and he had a normal range of motion of the affected knee. There was a history of injury to right knee three and half years back which was managed conservatively with medication and rest. On examination there was a nodular swelling on medial aspect of distal thigh about $3 \times 3 \mathrm{~cm}$ in size and tender to palpation. Blood parameters including the ESR were all normal. Imaging revealed an extra-articular, radio-dense, sessile bony lesion on the medial aspect of the distal femur. This lesion did not have any bony continuity with the native femoral cortex. (Figure 4) and (Figure 5)

At excision biopsy, the lesion was found to be an extra-articular cartilaginous mass about $1 \times 1 \mathrm{~cm}$ in size lying outside the periosteum of the distal medial femur. Histopathologic examination reported multiple nodules of cartilage embedded within soft tissue and foci of bone formation without any evidence of malignancy, thus a diagnosis of osteochondromatosis was reached. (Figure 6) and (Figure 7) 


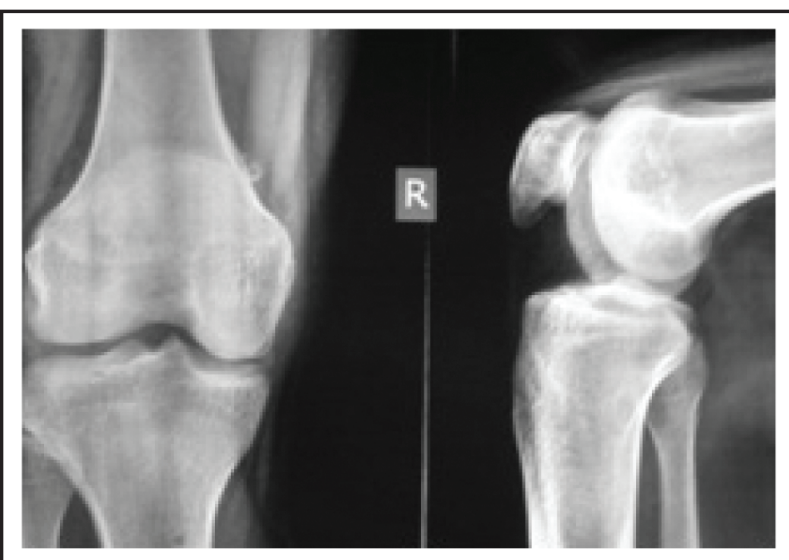

Figure 4. $\mathrm{X}$ ray of right knee AP and Lateral views showing a radiodense shadow on medial aspect of medial femoral condyle
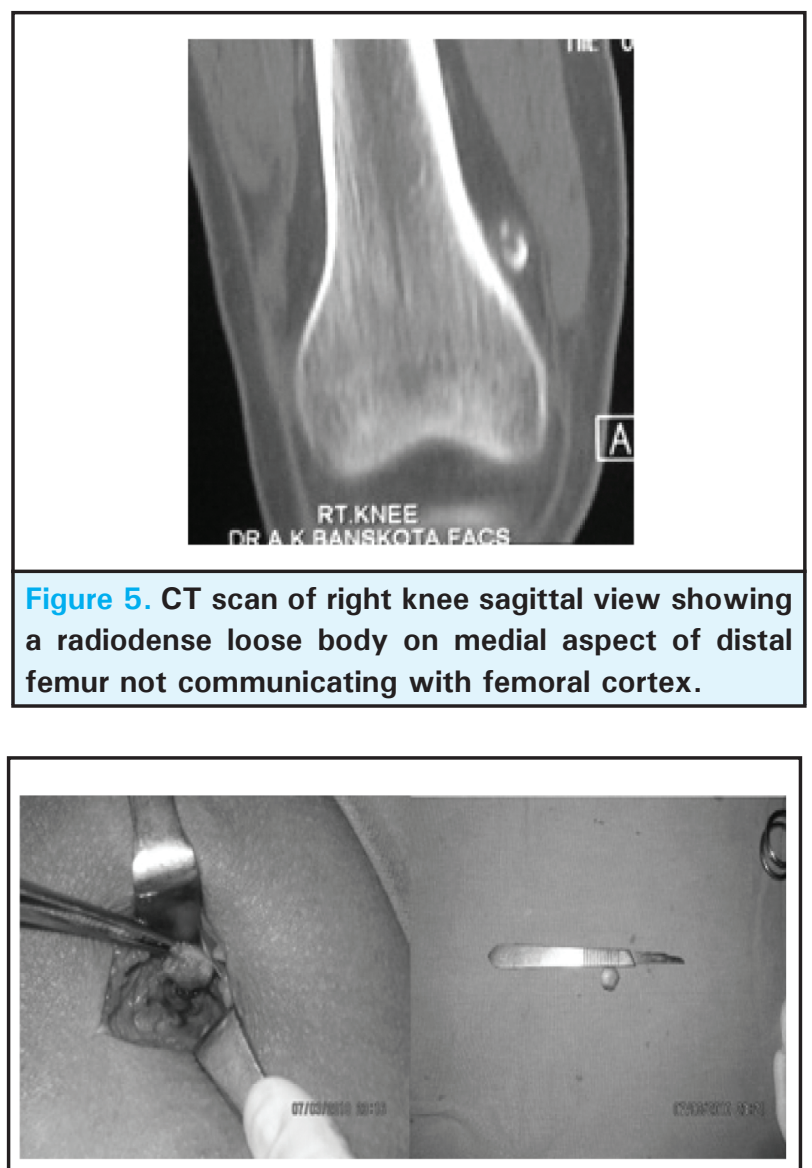

Figure 6. Intraoperative pictures showing a cartilagenous loose body removed from sheath of medial hamstring.

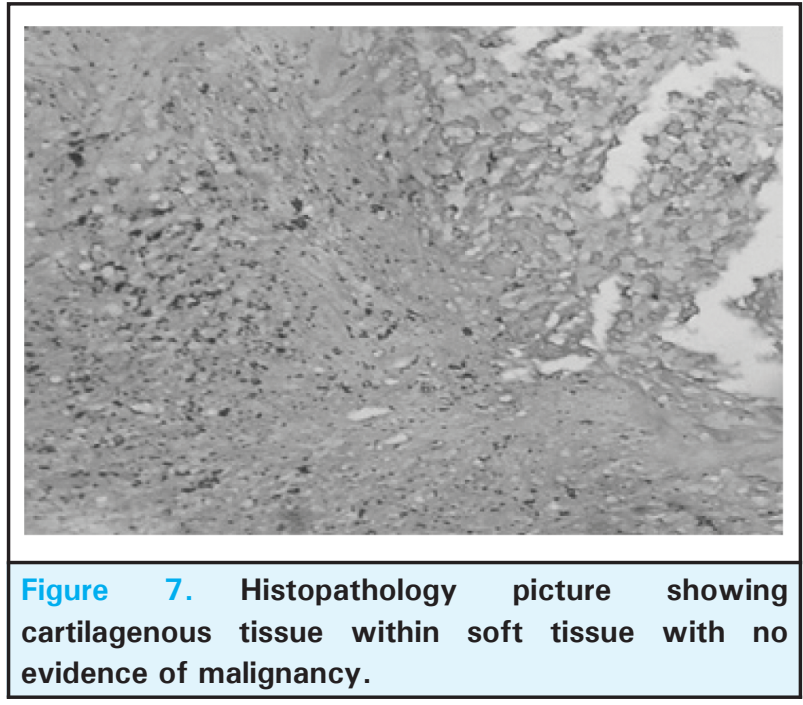

Post-operative recovery was uneventful and the patient was completely pain free on subsequent follow-ups.

\section{DISCUSSION}

Synovial chondromatosis is a rare condition in which islands of metaplastic cartilage develop within the synovium. ${ }^{1}$ It is an uncommon and benign disease of the synovium characterised by formation of multiple loose bodies, most of which subsequently become calcified and/or ossified. ${ }^{2}$ The occurrence rate is one per $100,000 \cdot{ }^{3}$ It is usually monoarticular, commonly affects the knee joint ${ }^{1,4}$, is twice as more common in men than in women ${ }^{2,5}$ usually presenting in their third to fifth decade of life. ${ }^{5}$ Extra-articular osteochondromatosis is extremely rare with few published reports in the literature. ${ }^{2}$ The true incidence of extra-articular synovial chondromatosis is unknown as proportion of patients will remain asymptomatic. ${ }^{6}$ It is thought to be a rare entity with few previous case reports. ${ }^{6}$ There are only four published cases of extra-articular synovial chondromatosis in prepubescent children. ${ }^{3}$ It arises from the synovium of tendon sheaths and bursae. ${ }^{2}$ Extraarticular synovial chondromatosis is usually solitary 7,8 and mostly involves the joints of the hands and feet. ${ }^{1,2,7}$

The cause of synovial chondromatosis is uncertain. ${ }^{2,4,5}$ There is metaplastic transformation of synovial cells into chondrocytes via an unknown stimulus. ${ }^{4,9}$ These chondrocytes become pedunculated and encrusted inside the synovium and eventually expelled into the joint as loose bodies ${ }^{4}$ which cause symptoms of pain, swelling and restricted range of motion. It may develop secondary to other conditions that can cause irritation of the synovium such as osteoarthritis, rheumatoid arthritis, osteochondritis dessicans and trauma. ${ }^{2}$

Histologically, it shows cellular atypia mimicking chondrosarcoma and malignant transformation is 
known to occur, albeit rarely ${ }^{5,8}$. Most lesions show a moderate nuclear atypia and double nucleated cells. ${ }^{1,5}$ In terms of cellular proliferative activity it appears to occupy an intermediate position between enchondroma and chondrosarcoma. ${ }^{5}$

The signs and symptoms of articular synovial chondromatosis are non specific. ${ }^{9}$ The presenting symptoms are pain, swelling and loss of joint movement. ${ }^{4}$ Milligram classified the disease into 3 phases:-active intrasynovial disease without loose bodies, active disease with loose bodies and multiple loose bodies but no intrasynovial disease..$^{2,4}$

Radiologically, synovial chondromatosis is characterised by the presence of multiple rounded loose bodies generally uniform in size and with variable degrees of calcification and/or ossification. ${ }^{2}$ Some lesions, therefore, may have radiolucent center due to incomplete calcification. ${ }^{2}$ CT scan may show erosion of adjacent joints. ${ }^{2}$
It is thus a benign condition with a strong tendency to eventual resolution. ${ }^{9}$ Surgical excision is warranted for symptomatic lesions and those causing symptoms secondary meniscal tears ${ }^{10}$. In patients with extraarticular bursal pathologies, the preferred surgical treatment is complete excision of involved bursa. ${ }^{4}$ Radiotherapy or chemotherapy have not been found to have a role in the treatment of synovial chondromatosis. ${ }^{4}$

Although a benign self-limiting process, recurrences after excision have been reported. ${ }^{2}$ Secondary degenerative arthritis is by far the most common complication of longstanding disease. ${ }^{2}$ Malignant transformation to chondrosarcoma is known complication but fortunately, rarely seen. ${ }^{2}$

\section{ACKNOWLEDGEMENT}

We would like to acknowledge Arjun Adhikari, IT Officer B \& B Hospital for helping us prepare this case report.

\section{REFERENCES}

1. Thomas E. Kaiser, John C. Ivins aand Krishnan K. Unni; Malingnant Transformation of Extra-articular Synovial Chondromatosis: Report of a Case Skeletal Radiology. 1980:5;223-6.

2. Fahad O. Alkubaidan, Eric J. Heffernan, Peter L. Munk; Extra-articular synovial chondromatosis in a prepatellar bursa, European Journal of Radiology Extra 2007:63:17-20.

3. Emily Ko, Errol Mortimer, Armando E Fraire; Extra-articular synovial chondromatosis: Review of Epidemiology, Imaging Studies, Microscopy and Pathogenesis, with a Report of a Additional Case in a Child; International Journal of Surgical Pathology 2004:12(3):273-80.

4. Hugh Mackenzie, Vivek Gulati, Samantha Tross; A rare case of swollen knee due to disseminated synovial chondromatosis: a case report, Journal of medical case reports 2010:4:113.

5. Richard I. Davis, Heather Foster, Ken Arthur, Sid Trewin, Peter W. Hamilton, Denis J. Biggart; Cell Proliferation Studies in Primary Synovial Chondromatosis, Journal of Pathology. 1998:184;18-23.
6. Millar TMS, Christofides A, Herlekar D, Courtman NH; Asymptomatic Extra-articular Synovial Osteochondromatosis of The Knee: Report of A Case; Journal of Orthopaedics 2007:4(3).

7. M. Demirhan, L. Eralp, A.C.Atalar; Synovial chondromatosis of the subchoracoid bursa

8. International Orthopedics(SICOT). 1999:3:358-60.

9. FH Sim, DC Dahlin and JC Ivins; Exrtra-articular Synovial chondromatosis

10. Journal of Bone and Joint Surgery Am.1977;59:492-5

11. T.E. Jeffreys, Oswestry; Synovial Chondromatosis, England Journal of Bone and Joint surgery 1967:49B(3):530-4.

12. Murat Bozkurt, Mahmut Ugurlu, Metin Dogan, Nihat Tosun; Synovial chondromatosis of four compartment of knee: medial and lateral tibiofemoral spaces, patellofemoral joint and proximal tibiofibular joint, Knee Surg Sports Traumatol Arthrose 2007:15:753-5. 